\title{
A Comparison of USP Paddle With Crescent- Shaped Spindle Dissolution Profiles
}

\author{
Suzanne Spagnoli ${ }^{1,2}$ and Marie Di Maso ${ }^{1}$
}

e-mail:suzanne_spagnoli@merck.com

\begin{abstract}
Dissolution profiles are used in making decisions on the quality of the product, the effect of critical processing parameters, the selection of the optimum formulation, and the determination of stability. The literature has demonstrated several initiatives to eliminate system artifacts not related to the drug product in dissolution testing. A proposed new spindle design was evaluated using various formulation challenges encountered in a pharmaceutical development group. The crescent-shaped spindle is in contact with the vessel bottom and the dosage unit, changing the hydrodynamics of the dissolution. Dissolution profiles using the crescent spindles were compared to those obtained using USP 2 paddles and related to in vivo behavior or quality control indicators. The practical use of the spindle for a GLP laboratory was also evaluated. This paper presents the results of these evaluations.

In two cases studied, pharmacokinetic data were related to the dissolution behavior. In both cases, the differences in the dissolution profiles for the variation in formulation or API properties obtained using the crescent spindles reflected the pharmacokinetic data, whereas this was true for only one case when using the USP 2 paddles. An evaluation of batch-to-batch reproducibility as a means of quality control resulted in a comparison of profiles using the USP 2 paddle versus the crescentshaped spindle. The crescent-shaped spindle provided data indicating that the dissolution profiles could discriminate differences in the manufactured product.

The design of the crescent-shaped spindle addressed the issue of poor hydrodynamics seen at low rotation for the paddles and allowed for easy installation into existing VanKel dissolution baths. Consistent positioning in the dissolution vessel was not easily achieved and resulted in higher variability. Consequently, the curvature of the crescent spindle should be fixed to ensure consistent contact with the vessel and the product. In order to be more widely accepted, calibration of the crescent-shaped spindle would require conformance to USP standards.
\end{abstract}

\section{Introduction}

$\mathrm{n}$ the pharmaceutical industry, dissolution testing is widely used as a quality control tool to ensure perfor-

mance of production lots and as a tool in the selection of formulations in development. In addition, evaluation of dissolution profiles as a surrogate for in vivo performance allows for the determination of bioequivalence of all formulations. The ability of dissolution to fulfill all of these roles is dependent on the reliability of the dissolution procedure. It has been well documented in the literature that the hydrodynamics of USP Apparatus 2 (paddle) may result in poor reproducibility and also poor variability in profiles not related to the formulation or in vivo characteristics $(1,2)$. Attempts to fully understand and prevent poor hydrodynamics at low paddle rotation speed has allowed for the advent of new dissolution techniques such as flat-bottom vessels or Peak ${ }^{\mathrm{TM}}$ vessels (3) to reduce cone formation, and only limited comparative data has been obtained on a new paddle design, the crescent-shaped spindle.

The rationale for the development of a crescent-shaped spindle is extensively documented in a report from the product designer and inventor, Dr. Saeed Qureshi (4). The crescent-shaped spindle design allows for intimate contact with the dosage form therefore eliminating coning and reducing vibration effects. Several evaluations of calibrator tablets, fast dissolving and extended-release tablets, and capsules have been performed $(4,5)$, and results have shown improved drug release with better product characterization.

When presented with the published data and asked to evaluate the crescent-shaped spindle, our focus and intent was to investigate in vitro-in vivo relationships as well as to evaluate the crescent-shaped spindle application as a batchto-batch quality control indicator. As an early phase development laboratory, our interest was in comparing animal or human bioavailability data with the dissolution profiles obtained with the crescent-shaped spindle and in comparing that data with previously obtained USP 2 paddle data. Evaluation of two cases was performed. In the first case, a formulation change was compared in vivo, and the results compared with USP 2 (paddle) dissolution profiles as well as crescentshaped spindle profiles. In the second case, the particle size effect on dissolution profiles was evaluated and compared to animal bioavailability data. As a quality control discriminator, several mis-manufactured batches were compared, along with a dual active batch. Throughout the evaluation, if sample was available, the dissolution profiles were also performed at low paddle rotation speed of $25 \mathrm{rpm}$ in order to compare and evaluate the potential for a single paddle rotation speed (6). 

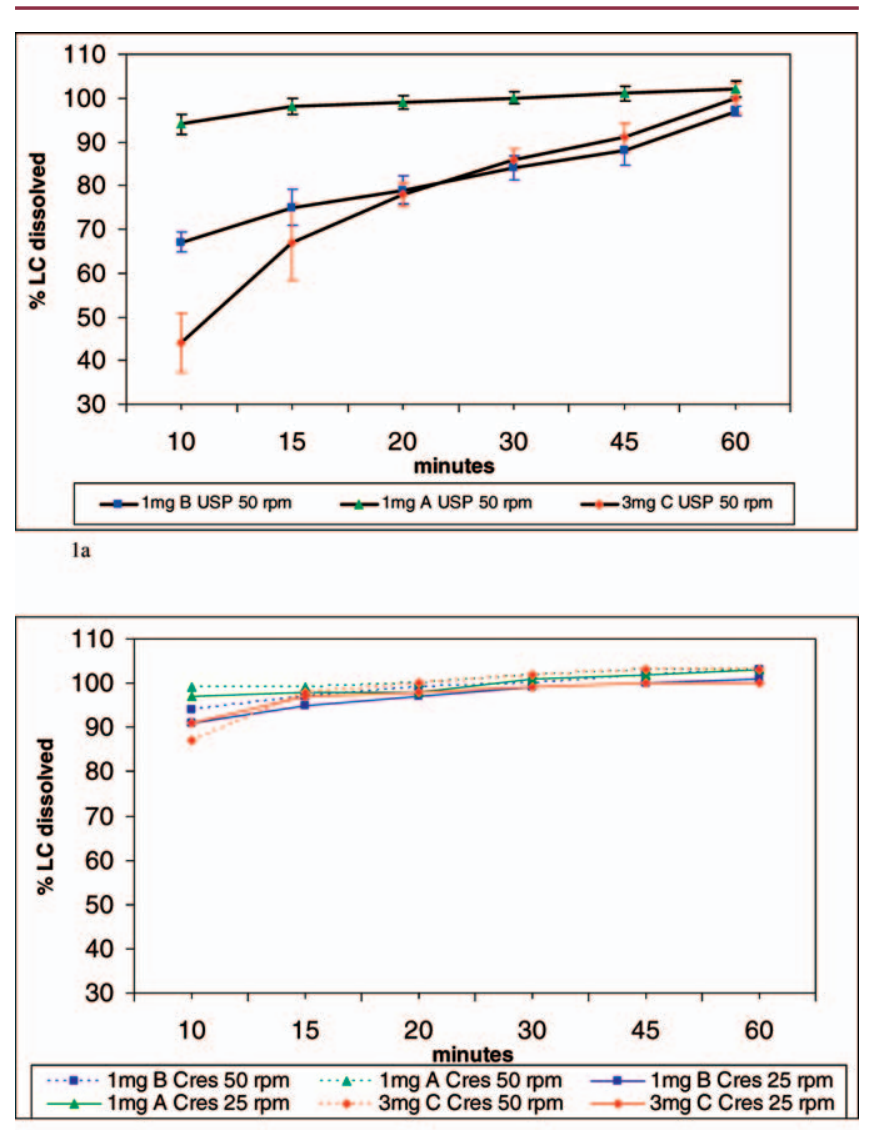

lb

Figure 1a: Comparison of Formulation A, Formulation B, and Formulation $C$ Using USP 2 Paddles (in $0.2 \%$ SDS)

Figure $1 b$ : Comparison of Formulation $A$, Formulation B, and Formulation $C$ Using Crescent-Shaped Spindle (in 0.2\% SDS)

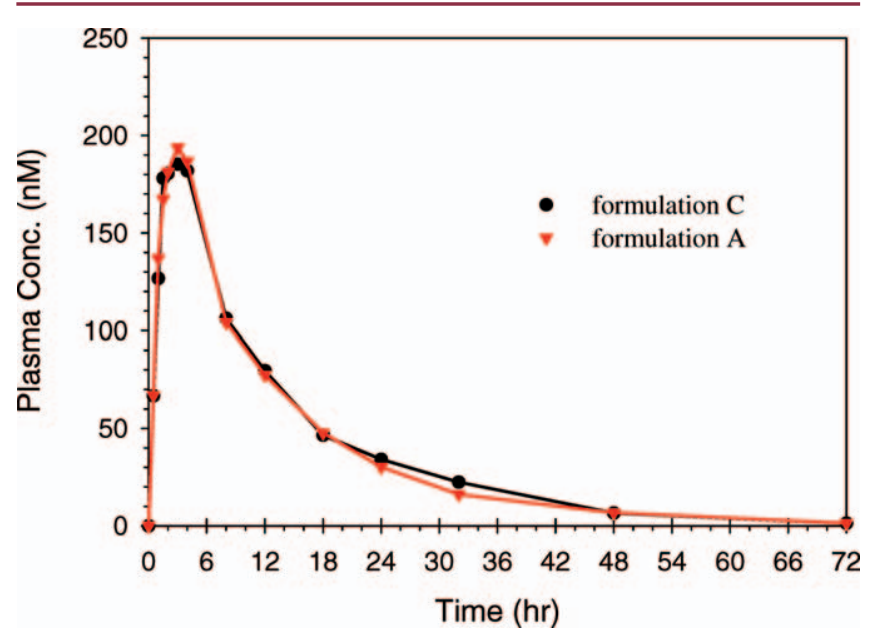

Figure 2: Mean Profiles $(\mathrm{N}=8)$ for Formulation $\mathrm{A}$ ( 3 tablets) and Formulation C (1 tablet) in Healthy Young Male Subjects

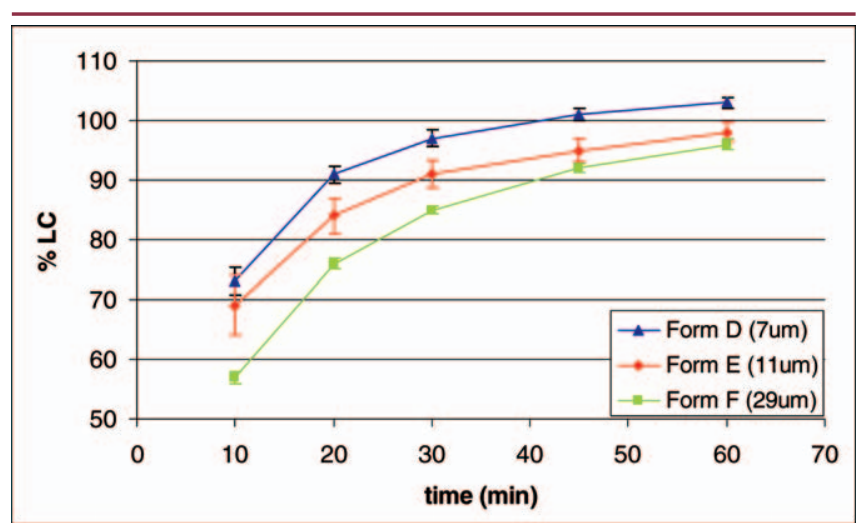

Figure 3: Particle Size Effect on Dissolution Rate for Formulations $D, E$, and F Using USP Paddles at $50 \mathrm{rpm}$ (2\% SDS)

\section{Results}

\section{Case 1: Formulation Change}

The first investigation involved comparison of two different immediate-release formulations of a given BCS Class IV drug substance, formulation A (uncoated) and formulation $B$ (coated, equivalent doses, different manufacturing procedure), to formulation $C$ ( 3 times the dose, equivalent manufacturing procedure to $B$ ). Formulation $A$ was the initial clinical dosage form and formulation $C$ was selected as the current clinical image, therefore a bioequivalence bridging study was required. A comparison of USP paddle profiles in Figure 1a shows a difference in dissolution profile between formulation $A$ versus formulation $B$ and $C$ reflective of the change in the manufacturing process. The USP paddle method for the initial 1-mg dose (formulation $A$ ) was developed using a paddle rotation of $50 \mathrm{rpm}$ until 45 minutes with an increase to $150 \mathrm{rpm}$ from 45 minutes to 60 minutes to ensure complete release of the active. When formulation $B$ and $C$ were evaluated using the same methodology, the dissolution profiles were depressed. The poor hydrodynamics of the USP paddle system at $50 \mathrm{rpm}$ affected the rate of release of the active in formulation $B$ and $C$, as shown by the slow release profile. Due to the small tablet size, the cone was not disturbed by the 50 -rpm paddle speed. Increasing the paddle speed to $75 \mathrm{rpm}$ was unlikely to significantly increase the dissolution rate for formulations $B$ and $C$ based

Table 1: Comparison of Dog Bioavailability for Formulations $D, E$, and $F$

(7 $\mu \mathrm{m}, 11 \mu \mathrm{m}$, and $29 \mu \mathrm{m}$, respectively)

\begin{tabular}{|llll|}
\hline Formulation & $D(7 \mu \mathrm{m})$ & $E(11 \mu \mathrm{m})$ & $\mathrm{F}(29 \mu \mathrm{m})$ \\
\hline $\mathrm{AUC}(\mu \mathrm{M} \cdot \mathrm{hr}) \pm S D$ & $0.942 \pm 0.747$ & $1.044 \pm 0.289$ & $0.578 \pm 0.228$ \\
\hline$C_{\max }(\mu \mathrm{M})$ & 0.33 & 0.26 & 0.11 \\
\hline $\mathrm{T}_{\max }$ & $1 \mathrm{hr}$ & $1 \mathrm{hr}$ & $2 \mathrm{hr}$ \\
\hline
\end{tabular}




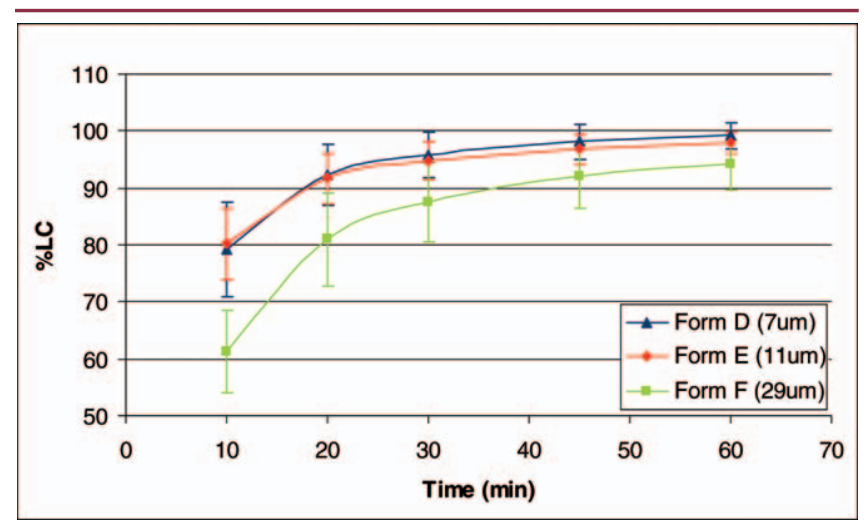

Figure 4: Crescent-Shaped Spindle Dissolution Profile for Formulations D (7 $\mu \mathrm{m}), E(11 \mu \mathrm{m})$, and $\mathrm{F}(29 \mu \mathrm{m})$ in $2 \%$ SDS at $25 \mathrm{rpm}$

on less than complete release achieved when ramped to 150 rpm at 45 minutes. When profiles were obtained using the crescent-shaped spindle, formulations $A, B$, and $C$ were equivalent, whether analyzed at $50 \mathrm{rpm}$, or slowed to $25 \mathrm{rpm}$ as shown in Figure 1b. The compound was not solubility limited as shown by the rapid release profiles in the crescent-shaped spindle experiments. The intimate contact of the crescent spindle with the tablet allowed for quick dissolution of the active ingredient with $>90 \%$ dissolved after 10 minutes, showing no difference between manufacturing process and tablet size. Since the clinical bioavailability data showed similar overlap of AUC as shown in Figure 2, the USP 2 method was overly discriminating, and the crescent-shaped spindle dissolution profile at either $25 \mathrm{rpm}$ or $50 \mathrm{rpm}$ provided a rapid dissolution procedure without being overly discriminating relative to in vivo behavior.

\section{Case 2: API Particle Size Effect}

Another compound, a BCS Class II, was investigated whereby the differences in active pharmaceutical ingredient (API) particle size affected bioavailability and conventional USP paddle dissolution profiles. The USP dissolution profiles in $2 \%$ sodium dodecyl sulfate (SDS) at $50 \mathrm{rpm}$ are

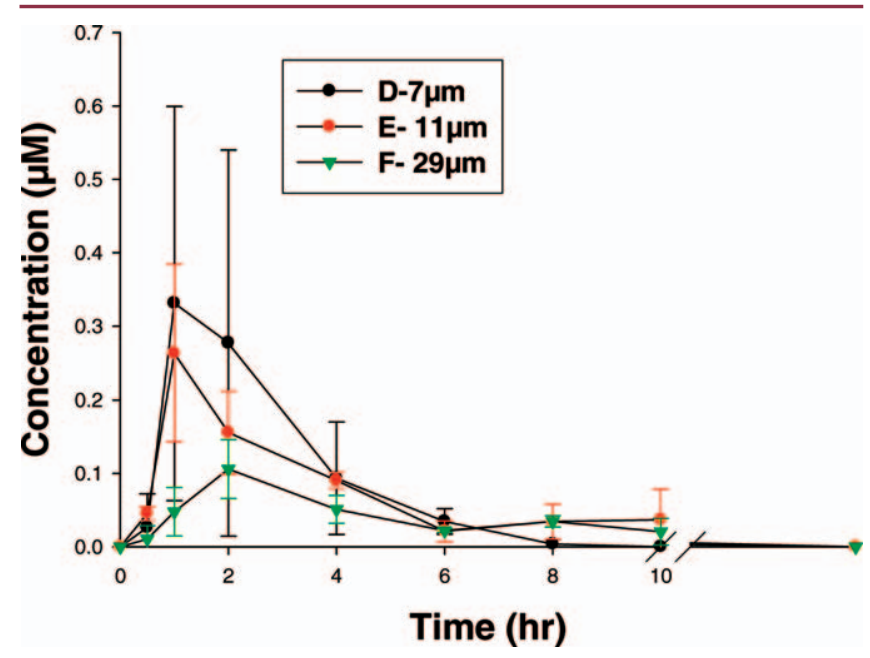

Figure 5: Plasma Levels of Formulations D, E, and F Given in Dogs (20-mg tablet) given in Figure 3 for formulations $\mathrm{D}(7 \mu \mathrm{m}), \mathrm{E}(11 \mu \mathrm{m})$, and $\mathrm{F}$ $(29 \mu \mathrm{m})$; the tablets are equivalent in size and shape. The dissolution profiles were also obtained using the crescentshaped spindle at $25 \mathrm{rpm}$ in $2 \%$ SDS (Figure 4). The dissolution profile at $50 \mathrm{rpm}$ using the crescent-shaped spindles was not pursued since the $25 \mathrm{rpm}$ showed trends consistent with dog bioavailability data.

The in vitro profiles for formulations $\mathrm{D}, \mathrm{E}$, and $\mathrm{F}$ obtained using USP paddles and crescent spindles correlated well with pharmacokinetic data obtained in dogs. The larger particle size API (formulation F) showed slower rates of dissolution in both cases and had depressed $C_{\max }$ and lower AUC as shown in Table 1 and Figure 5. A comparison of the bioavailability for formulations $D$ and $E$ showed similar $A U C$ and $T_{\text {max }}$, which is reflected by the crescent-shaped spindle dissolution profile in Figure 4. Since the 29- $\mu \mathrm{m}$ particle size API had slower dissolution profiles in both the USP paddle and crescentshaped spindle configurations, it indicates that the in vitro dissolution rate is driven by intrinsic dissolution where the physical attributes of the API control the solubilisation of the drug. Although faster dissolution profiles were obtained with the crescent-shaped spindle, both dissolution tests were able to discriminate for the formulation differences showing the reduced $\mathrm{pK}$ in dogs.

\section{Case 3: Formulation Quality Control Evaluation: Dual Active Tablets}

Another aspect of the importance of dissolution testing involves monitoring formulations for changes in manufacturing and excipient ratios. In order to evaluate the potential of the crescent-shaped spindle, control and deliberately mismanufactured tablets were evaluated.

The investigation involved a dual active BCS Class II tablet that was intentionally mis-manufactured by altering the excipient levels and by modifying compression strength. The current method required a $900-\mathrm{mL}$ volume of $0.5 \%$ SDS with USP 2 paddles rotated at $75 \mathrm{rpm}$ in an attempt to prevent cone formation. Since no bioequivalence data was available, a direct comparison of profiles at the same rotation speed was conducted. The dual active tablets (control formulation $\mathrm{G}$ and mis-manufactured formulation $\mathrm{H}$ ) were analyzed with the USP 2 paddle and compared to the crescent-shaped spindle at $75 \mathrm{rpm}$ in $900 \mathrm{~mL}$ of $0.5 \%$ SDS as shown in Figure $6 a$, b. The dissolution profiles obtained at $75 \mathrm{rpm}$ showed that the mismanufactured tablets have a slower release profile than the control tablets. An additional experiment was performed at $50 \mathrm{rpm}$ for both the paddle and the crescent-shaped spindle. The data are shown in Figure $7 a$, b. Since the rate of release for the USP 2 paddle data is much slower at $50 \mathrm{rpm}$, it indicates that there is inherent variability due to coning at $50 \mathrm{rpm}$. Since the crescent spindles prevent any coning, the rate of dissolution is faster than conventional USP paddle data obtained in the same media.

To further understand the impact of coning in the USP 2 apparatus, the control and mis-manufactured tablets were analyzed using Peak ${ }^{\mathrm{TM}}$ dissolution vessels $(0.2 \%$ SDS at 50 rpm), and the profiles are shown in Figure 8. 


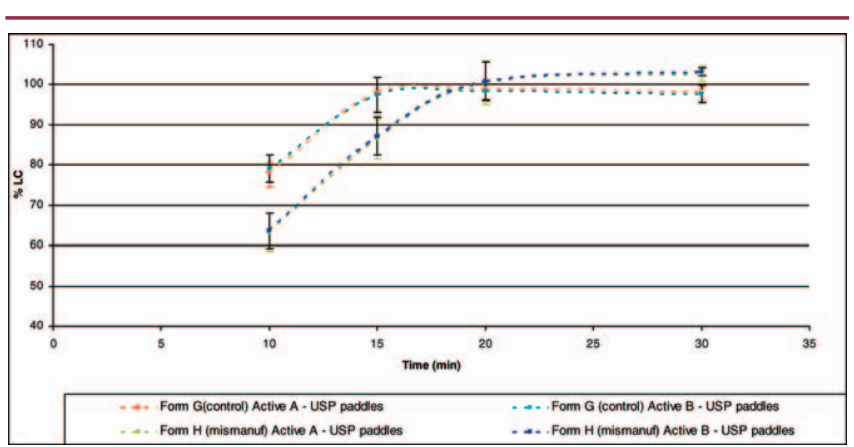

Figure 6a:Dissolution Profiles for Dual Active Tablet at $75 \mathrm{rpm}$ Using USP 2 Paddle

Since the dissolution profile in the Peak ${ }^{\mathrm{TM}}$ vessel at $50 \mathrm{rpm}$ is faster than that obtained using regular vessels (Figure 7a,b), it confirms that coning is an issue for this product. An interesting note is that the rate of release of active using the Peak $^{\mathrm{TM}}$ vessel is more consistent with crescent-shaped spindle data, which would support both methods as effective means of reducing cone formation. The USP 2 paddle data at $75 \mathrm{rpm}$ has dissolution profiles that are much slower when compared to peak or crescent spindle data, indicating that coning is still an issue at $75 \mathrm{rpm}$. As a quality control tool, the crescentshaped spindle can show differences in dissolution profiles at the early time point of 10 minutes, but with comparison to a $Q$ value at either 15 or 20 minutes, could not discriminate between control and mis-manufactured tablets under the current dissolution conditions. The in vivo performance of the mis-manufactured batches was not determined.

\section{Case 4: Formulation Quality Control Evaluation: Effect of Tablet Hardness and Disintegrant Levels}

Another BCS Class II API was analyzed in order to evaluate the potential for the crescent-shaped spindle as a quality control dissolution tool. Two worst-case core formulations were monitored: lowest dose (formulation I) and highest dose (formulation $\mathrm{K}$ ), each with no disintegrant across a low, target, and high compression range. The effect on dissolution profile of the core tablets was monitored using conventional USP 2 paddle and crescent-shaped spindle. In addition, high-dose tablets with varying levels of disintegrant (at target hardness) were film coated (formulation M)

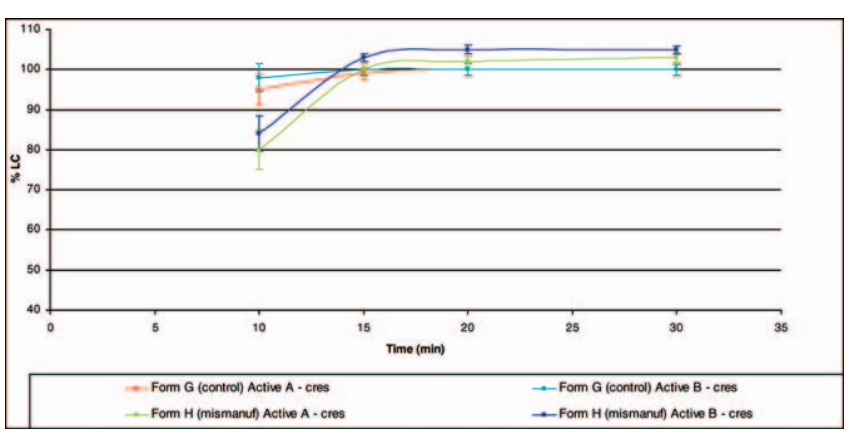

Figure 6b: Dissolution Profiles for Dual Active Tablet at $75 \mathrm{rpm}$ Using Crescent-Shaped Spindle

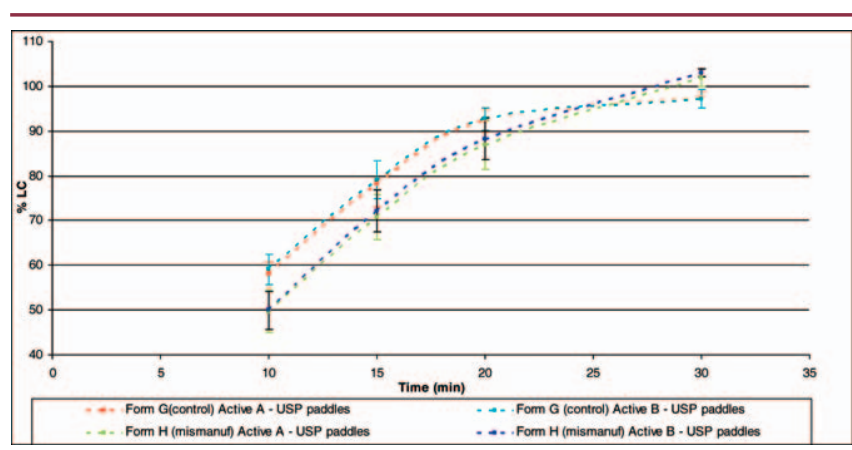

Figure 7a: Dissolution Profiles for Dual Active Tablet at $50 \mathrm{rpm}$ Using USP 2 Paddle

and similarly analyzed. The data are summarized in Figures $9,10,11$, and 12.

The crescent spindle dissolution profiles had significant variability in the testing results as shown by the large error bars in Figures 9-11. When no disintegrant was present in the tablet, the effect on the dissolution profile was more significant for the high-dose tablets. The crescent spindles rotated at $25 \mathrm{rpm}$ would provide profiles indicating that core tablets manufactured without disintegrant would not meet Stage 1 testing with current specifications set at 15 minutes. This assumes that the variability between crescentshaped spindles improves. Similarly with the final filmcoated tablets, the crescent-shaped spindle dissolution profiles (Figure 11) would provide an indication that samples without disintegrant would not meet the current $Q$ specification of $80 \%$ at 15 minutes but could potentially meet Stage 2 testing.

The USP 2 paddle data demonstrates that at the current method parameters of $75 \mathrm{rpm}$, the specification of $>80 \%$ dissolved at 15 minutes is satisfied for either the low dose or the high dose core tablets (USP 2 paddle data not shown). Some discrimination was noted for the final film-coated tablet with no disintegrant as shown in Figure 12, although Stage 2 testing requirements would be met. Note reduced variability at each sampling point using the USP 2 paddle as highlighted in Figure 12 when compared to crescent paddle data, which may be related to the robustness of the crescent-shaped spindle.

In general, the crescent-shaped spindle dissolution profiles were more sensitive in discerning the level of disin-

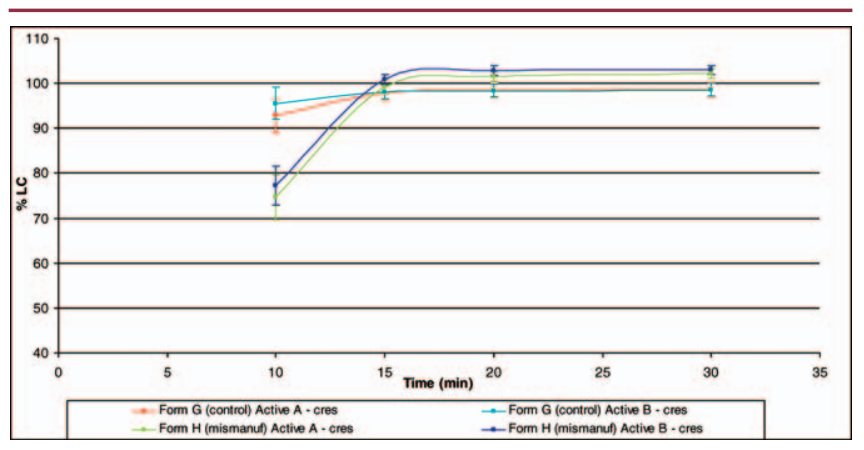

Figure 7b: Dissolution Profiles for Dual Active Tablet at $50 \mathrm{rpm}$ Using Crescent-Shaped Spindle 


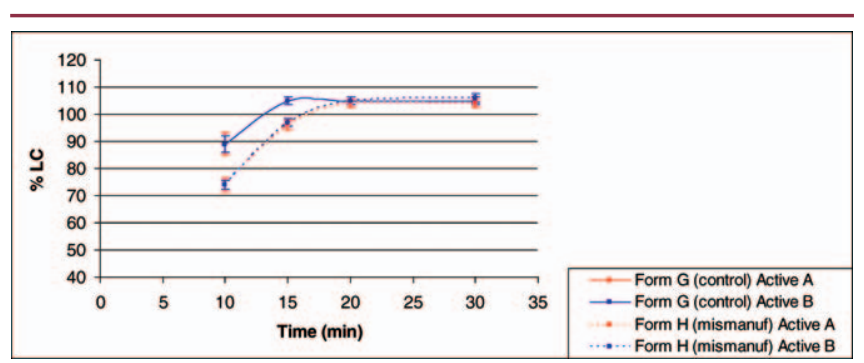

Figure 8: Dissolution Profiles for Dual Active Tablets (control and mis-manufactured) at $50 \mathrm{rpm}$ Using Peak ${ }^{\mathrm{TM}}$ Vessels

tegrant in the tablets, especially for the high-dose, larger tablets. An important note is that when no disintegrant is present, the dissolution profiles are slower at all hardnesses. The significance of this slower rate is unknown without in vivo data, which was not available for this study. Hypothetically, if the in vivo data supported poor bioavailability for tablets with no disintegrant, then the crescent spindle could be used as a quality control tool with the appropriate specifications selected

\section{Evaluation of Hydrodynamics}

To ensure robustness of the spindle in a working laboratory where transfer to other dissolution baths and manipulations are frequent, a means of consistently positioning the spindle would be required to reduce the variability associated with the test. To address some of the issues associated with the crescent-spindle design, the existing USP paddle was lowered such that it was positioned approximately 5 $\mathrm{mm}$ from the vessel bottom. The rationale was to reproduce the hydrodynamics of the crescent-shaped spindle using an existing approved apparatus and paddle. The USP paddle was adjusted to sit as low as possible in the vessel. Due to the design of the vessel and the width of the paddle, the paddle could not sit on the bottom of the vessel. Evaluation of formulations D and F (Case 2) using the lowered USP 2 paddles rotated at $25 \mathrm{rpm}$ produced very slow rates of release $(<50 \%$ released at 60 minutes) indicating that lowered USP paddles were unable to match the dissolution profiles achieved using the crescent paddle at $25 \mathrm{rpm}$ (Figure 4). Increasing the lowered paddle speed to $50 \mathrm{rpm}$ in

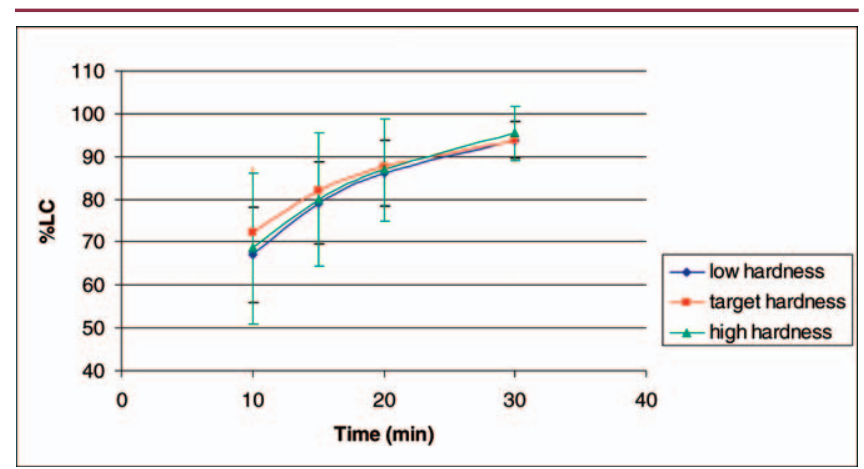

Figure 9: Dissolution Profile for Mis-Manufactured Core Tablets (formulation I: low dose/no disintegrant) Using Crescent-Shaped Spindles $(900 \mathrm{~mL}$ $0.01 \mathrm{~N} \mathrm{HCl}$ at $25 \mathrm{rpm}$ )

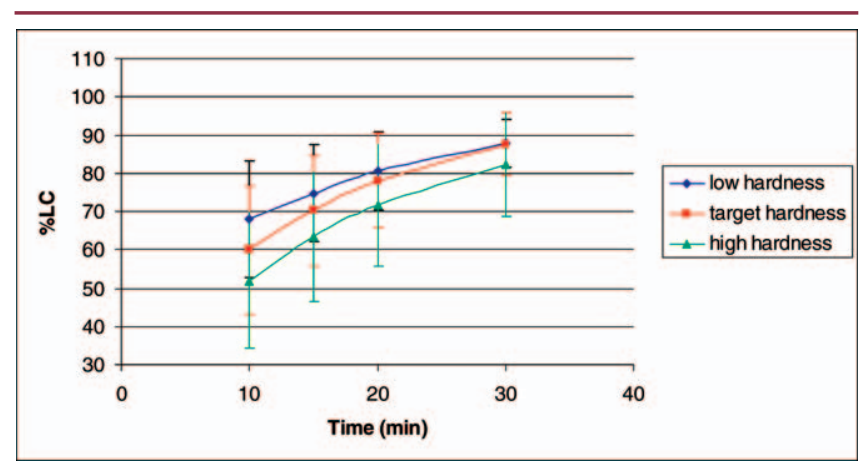

Figure 10: Dissolution Profile for Mis-Manufactured Core Tablets (formulation K: high dose/no disintegrant) Using Crescent-Shaped Spindles $(900 \mathrm{~mL}$ $0.01 \mathrm{~N} \mathrm{HCl}$ at $25 \mathrm{rpm}$ )

an attempt to generate improved hydrodynamics did not mimic the crescent data. The data in Figure 13 resembles that for the conventional USP paddle at $50 \mathrm{rpm}$ (Figure 3). Lowering the USP paddle and rotating it at $25 \mathrm{rpm}$ had minimal effect on the hydrodynamics of the media and could not reproduce the actual tablet movement achieved with the crescent-shaped spindle design and the hydrodynamics achieved with the larger surface area spindle rotating on the bottom of the vessel.

\section{Conclusion}

In the cases evaluated, the crescent-shaped spindle addressed the poor hydrodynamics of the USP 2 dissolution apparatus. The in vitro dissolution profiles reflected the drug product and correlated well with in vivo data. The crescentshaped spindle could provide insight into the effect of API particle size or formulation changes on the biological behavior. Additional in vitro-in vivo evaluations are necessary to further support the use of crescent spindle dissolution as an exploratory tool in early development.

Additionally, the acceptance of the crescent-shaped spindle by the USP for routine dissolution testing would require improvements in the robustness of the crescentshaped paddle curvature, reproducible positioning in the vessel, maintenance of bristles, and acceptable calibration in order to reduce variability in the measurements and allow for consistent comparisons. Since the Peak ${ }^{\mathrm{TM}}$ vessel, which

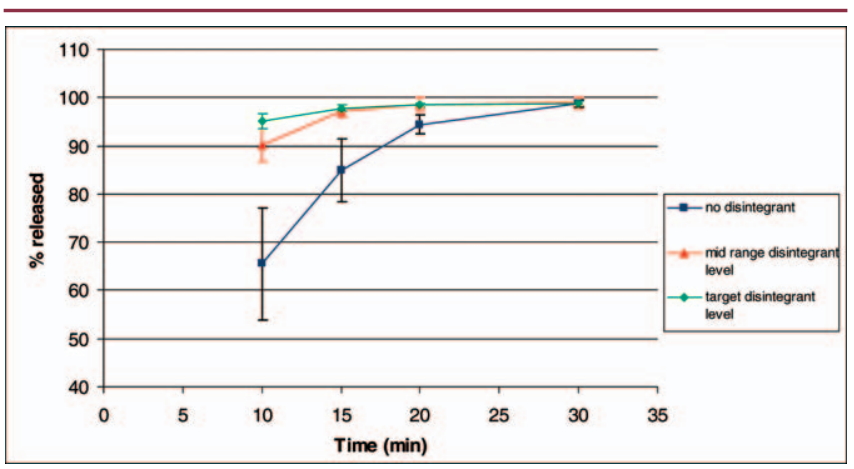

Figure 11: Dissolution Profile of Film Coated Mis-Manufactured Tablets (formulation M: high dose/target hardness/varying disintegrant level) Using Crescent-Shaped Spindle (900 mL $0.01 \mathrm{~N} \mathrm{HCl}$ at $25 \mathrm{rpm}$ ) 


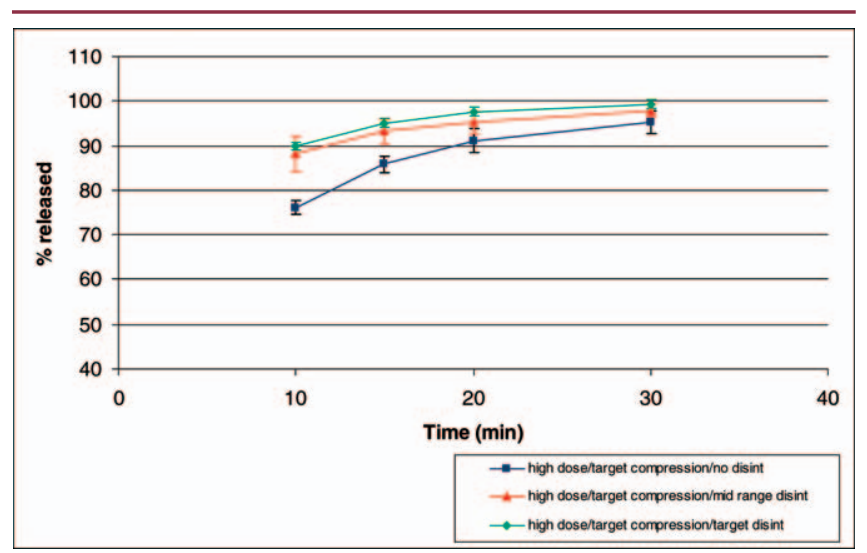

Figure 12: Dissolution Profile of Film Coated Mis-Manufactured Tablets (formulation M: target hardness/varying disintegrant level) Using USP 2 Paddles (900 mL $0.01 \mathrm{~N} \mathrm{HCl} \mathrm{at} 75 \mathrm{rpm}$ )

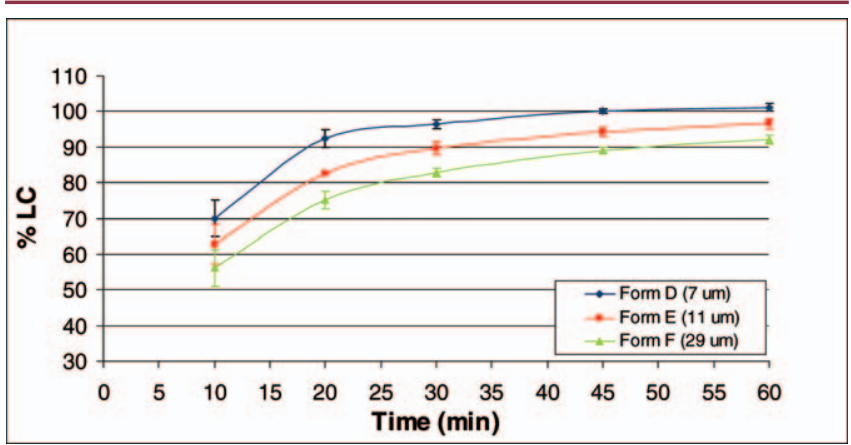

Figure 13: Lowered USP Dissolution Paddle Profile for Formulation D (7 $\mu \mathrm{m})$ and $F(29 \mu \mathrm{m})(2 \%$ SDS, $50 \mathrm{rpm})$

satisfies the aforementioned criteria, still has not gained world-wide acceptance, the crescent-shaped spindle will face many of the same challenges.

\section{Acknowledgements}

The authors would like to thank Dr. Saeed Qureshi (Therapeutic Products Directorate, Canada) for the crescent-shaped spindles, Dr. Elizabeth Kwong (Merck Frosst) for her insights and discussions, and Maria Marcantonio, Tony Lee, and Rahul Chitrapu for performing many of the dissolution analyses.

\section{References}

1. Kukura, J.; Arratia, P. E.; Szalai, E. S.; Muzzio, F. J. Engineering Tools for Understanding the Hydrodynamics of Dissolution Tests. Drug Dev. Ind. Pharm. 2003, 29, 231-239.

2. McCarthy, L. G.; Bradley, G.; Sexton, J. C.; Corrigan, O. I.; Healy, A. M. Computational Fluid Dynamics Modeling of the Paddle Dissolution Apparatus: Agitation Rate, Mixing Patterns, and Fluid Velocities. AAPS PharmSciTech 2004, 5 (2), Article 31.

3. Mirza, T.; Joshi, Y.; Liu, Q.; Vivilecchia, R. Evaluation of Dissolution Hydrodynamics in the USP, Peak ${ }^{\mathrm{TM}}$ and
Flat-Bottom Vessels Using Different Solubility Drugs. Dissolution Technologies 2005, 12 (1), 11-16.

4. Qureshi, S. A. A New Crescent-Shaped Spindle for Drug Dissolution Testing-But Why a New Spindle? Dissolution Technologies 2004, 11 (4), 13-18.

5. Qureshi, S. A. Improved Drug Dissolution and Product Characterization Using a Crescent-Shaped Spindle. J. Pharm. Pharmacol. 2004, 56 (9), 1135-1141.

6. Qureshi, S. A. Choice of Rotation Speed (rpm) for BioRelevant Drug Dissolution Testing Using a CrescentShaped Spindle. Eur J. Pharm. Sci. 2004, 23 (3), 271-275. 